\title{
Assessment of cognitive function in female rheumatoid arthritis patients: associations with cerebrovascular pathology, depression and anxiety
}

\author{
Csaba Oláh ${ }^{1} \cdot$ Zsófia Kardos $^{2} \cdot$ Mónika Andrejkovics $^{3,4}$ - Enikő Szarka ${ }^{1} \cdot$ Katalin Hodosi ${ }^{5} \cdot$ Andrea Domján $^{5}$. \\ Mariann Sepsi ${ }^{6}$ - Attila Sas ${ }^{7} \cdot$ László Kostyál $^{6} \cdot$ Katalin Fazekas $^{2}$. Ágnes Flórián ${ }^{2} \cdot$ Katalin Lukács $^{2}$. Ágnes Miksi ${ }^{2}$. \\ Zsuzsanna Baráth ${ }^{2} \cdot$ György Kerekes $^{8} \cdot$ Márta Péntek $^{9,10} \cdot$ Attila Valikovics $^{7} \cdot$ László Tamási $^{2} \cdot$ Dániel Bereczki $^{11}$. \\ Zoltán Szekanecz ${ }^{5}$
}

Received: 21 July 2019 / Accepted: 17 September 2019 / Published online: 25 September 2019

(C) The Author(s) 2019

\begin{abstract}
We assessed cognitive function of female rheumatoid arthritis (RA) patients and analyze the determinants, with special focus on cerebrovascular morphology. Sixty methotrexate (MTX-) or biologic-treated RA patients and 39 healthy controls were included in a cross-sectional study. Smoking habits, alcohol intake and time spent in education were recorded. Standard measures were performed to assess cognitive function (Montreal Cognitive Assessment, MOCA; Trail Making Test, TMT; Victoria Stroop Test, VST; Wechsler Adult Intelligence Scale, WAIS; Benton Visual Retention test, BVRT), depression (Beck Depression Inventory, BDI), anxiety (State-Trait Anxiety Inventory, STAIT/S) and general health status (Short Form 36, SF-36). Mean disease activity (28-joint Disease Activity Score, mDAS28; erythrocyte sedimentation rate, mESR; C-reactive protein, $\mathrm{mCRP}$ ) of the past 12 months was calculated; anti-cyclic citrullinated peptide (CCP) and rheumatoid factor (RF) were assessed. Cerebral vascular lesions and atrophy, carotid intima-media thickness (cIMT) and plaques, as well as median cerebral artery (MCA) circulatory reserve capacity (CRC) were assessed by brain magnetic resonance imaging (MRI), carotid ultrasound and transcranial Doppler, respectively. Cognitive function tests showed impairment in RA vs controls. Biologic- vs MTX-treated subgroups differed in TMT-A. Correlations were identified between cognitive function and depression/anxiety tests. WAIS, STAIS, STAIT and BDI correlated with most SF-36 domains. Numerous cognitive tests correlated with age and lower education. Some also correlated with disease duration, mESR and mDAS28. Regarding vascular pathophysiology, cerebral vascular lesions were associated with VST-A, carotid plaques with multiple cognitive parameters, while MCA and CRC with MOCA, BVRT and BDI. RA patients have significant cognitive impairment. Cognitive dysfunction may occur together with or independently of depression/anxiety. Older patients and those with lower education are at higher risk to develop cognitive impairment. Cognitive screening might be a useful tool to identify subgroups to be further investigated for cerebrovascular pathologies.
\end{abstract}

Keywords Rheumatoid arthritis · Cognitive dysfunction · Cerebrovascular disease · Transcranial Doppler · Carotid artery · Methotrexate $\cdot$ Biological therapy

\section{Introduction}

Rheumatoid arthritis (RA) may involve the vascular and nervous systems [1,2]. There is increased risk of ischemic stroke, especially in elderly RA patients with long-standing

Zoltán Szekanecz

szekanecz.zoltan@med.unideb.hu

Extended author information available on the last page of the article disease [3]. We have recently reported abnormalities in the cerebral circulation in transcranial Doppler (TCD) and vascular lesions by brain MRI in RA [4]. Functional MRI revealed the association of inflammation with brain structure and connectivity in RA [5]. Depression and anxiety are present in two-third of RA patients [6-8].

Cognitive function includes orientation, attention/concentration, judgment/problem solving, memory, verbal, visual/spatial and executive functions [9, 10]. RA patients may significantly underperform on cognitive function tests 
compared to controls [9,11-15]. Even mild impairment may influence the functional capacity and quality of life of RA patients $[9,13,16,17]$. It affects reactivity to pain, compliance to and effectiveness of therapy [9].

In general, both inflammation itself that may also affect the brain $[5,9,17]$ and accelerated atherosclerosis and increased cardiovascular (CV) pathology also driven by systemic inflammation [13, 17-19] may cause cognitive impairment in RA. Pain, stress, fatigue, sleep disturbances, depression and anxiety may also be involved in RA-associated cognitive decline [7, 9, 13]. Methotrexate (MTX) and corticosteroids have been associated with cognitive impairment $[9,20,21]$. As inflammation is involved in cognitive dysfunction in RA, the control of disease activity may be beneficial for the patients [22, 23].

We wished to assess cognitive function in a subset of Hungarian patients with RA compared to healthy controls using multiple standardized cognitive tests in parallel with imaging assessment of cerebrovascular morphology. Regarding explanatory factors, we focused on six major areas: some demographic parameters (age, educational level), lifestyle factors (smoking, alcohol intake), disease characteristics (duration, seropositivity, activity), treatment subgroups (MTX, biologic), physical and mental health status (SF36), mood problems (depression, anxiety) and cerebrovascular morphology [carotid atherosclerosis, cerebrovascular reserve capacity (CRC) of median cerebral artery (MCA), brain MRI]. The novelty of our study is that we used multiple tests of cognitive function and we associated these tests with cerebrovascular imaging.

\section{Patients and methods}

\section{Patients and controls}

Sixty female RA patients undergoing regular follow-ups at the Borsod County Teaching Hospital, Miskolc were selected. The inclusion criteria were female patients aged 18 or over; definitive diagnosis of RA; stable dose of MTX, corticosteroids and biologics for at least 6 months prior to the study; and informed consent. The exclusion criteria were pregnancy and/or breast feeding; any previous vascular events including cerebrovascular diseases, as well as mental disorders; history of skull trauma; known depression and other mood disorders; implanted metal; claustrophobia. Twenty patients were biologic naive. They had been receiving MTX for a mean 7.2 \pm 4.9 (range $2-15$ ) years in an average dose of $14.1 \pm 4.4$ (range 5-25) mg per week. We selected 40 female patients receiving biologics [20 infliximab (IFX) and 20 tocilizumab (TCZ)] as first-line biologic treatment at the time of the survey, in combination with MTX, for a mean duration of $4.0 \pm 2.0$ (range 1-10) years.
We selected intravenously administered biologics to increase compliance and therapy control. Moreover, we had more time to perform the psychologic and other diagnostic tests. The doses of MTX and biologics had been stable for at least 3 months prior to the study. Disease activity (DAS28-ESR) was determined in all RA patients every 3 months; thus, the mean values of the past 5 visits ( 12 months) were calculated and used in our analyses (Table 1).

For this study, a convenience sample of 39 healthy female individuals was chosen as the control group. These controls participated in a carotid ultrasound screening program carried out in the Borsod County region by some of the authors. Controls had the same inclusion and exclusion criteria as the RA patients. The age of controls (60.2 \pm 6.7 years) was not significantly different from that of RA patients (Table 1). As normative data with the Short Form 36 (SF-36) general health status measure are available for the Hungarian general population, RA patients' SF-36 scores were compared to the gender- and age-matched population norm [24].

We assessed smoking habits, alcohol consumption during the past 2 years and the number of years in education (school years) by a questionnaire (Table 1 ).

Ethical approval (No. 1046-63/2015) was obtained from the Regional/Institutional Review Board of Miskolc University. All patients and controls signed informed consent. The study was performed according to the Declaration of Helsinki.

\section{Tests of cognitive function and depression/anxiety}

The following standardized tests also translated to Hungarian were used (Table 2) [25]:

Mental capabilities The Montreal cognitive assessment (MOCA) test is a screening tool to determine mental capabilities in general and for determining mild cognitive impairment. The higher values indicate better cognitive function (range 0-30); a score of $\geq 26$ is considered as normal [26].

Attention/concentration The Digit Symbol subtest of the Wechsler Adult Intelligence Scale (WAIS) provides information on visuo-perceptual functions, fine visual-motor dexterity and speed. The number of correct symbols reproduced within the time limit is measured. Numbers are then converted to $\mathrm{Q}$ points (age-adjusted standard scores in the Hungarian version) $[27,28]$. Trail making test (TMT) measures the visual attention, speed of visual processing, mental flexibility, and executive functions. In part A of the TMT (TMT-A) assessing visuomotor processing speed, numbers are presented on a page in random array. The patient is instructed to connect the numbers in ascending order as quickly as possible. Part B (TMT-B) determines mental flexibility and measures the control and executive function of the frontal lobe of the brain. Here, both numbers and letters are on the sheet and the examinee must alternate connecting 
Table 1 General characteristics and laboratory markers of assessed RA patients and controls

\begin{tabular}{lllll}
\hline & RA (total) & RA (MTX) & RA (biologic) & Controls \\
\hline$n$ & 60 & 20 & 40 & 39 \\
Age (years) & $60.7 \pm 9.5$ & $61.3 \pm 9.1$ & $60.4 \pm 9.8$ & $60.2 \pm 6.7$ \\
Sex (females) & 60 & 20 & 20 & 39 \\
Disease duration (years) & $11.6 \pm 7.6$ & $11.8 \pm 8.6$ & $11.6 \pm 7.1$ & - \\
RF positivity (\%) & $70 \%$ & $82 \%$ & $66 \%$ & - \\
Anti-CCP positivity (\%) & $65 \%$ & $75 \%$ & $64 \%$ & - \\
Patients with erosions on hand/ & $54 / 60(90)$ & $18 / 20(90)$ & $36 / 40(90)$ & \\
feet X-ray (\%) & & & & - \\
MTX duration (years) & $6.7 \pm 4.1$ & $7.2 \pm 4.9$ & $6.4 \pm 3.6$ & - \\
MTX dose (mg/week) & $14.9 \pm 5.5$ & $14.1 \pm 4.4$ & $15.3 \pm 5.9$ & - \\
Biologic duration (years) & $4.0 \pm 2.0$ & - & $4.0 \pm 2.0$ & - \\
mDAS28-ESR & $2.50 \pm 0.87$ & $2.89 \pm 0.80$ & $2.30 \pm 0.78$ & - \\
mESR (mm/h) & $17.7 \pm 15.9$ & $20.3 \pm 16.6$ & $16.4 \pm 15.7$ & $12.3 \pm 1.8$ \\
hsCRP (mg/l) & $5.2 \pm 7.7$ & $8.5 \pm 10.9$ & $3.5 \pm 4.7$ & $3 / 39(7.7)$ \\
Time at school (years) & $11.4 \pm 3.0$ & $12.5 \pm 2.7$ & $10.8 \pm 3.0$ & $10 / 39(25.6)$ \\
Smoking (\%) & $10 / 60(16.6)$ & $5 / 20(25.0)$ & $5 / 40(12.5)$ & $37(95)$ \\
Alcohol consumption (\%) & $5 / 60(8.3)$ & $2 / 20(10.0)$ & $3 / 40(7.5)$ & $3 / 40(7.5)$ \\
Current workability (\%) & $9 / 60(15)$ & $6 / 20(30)$ & & \\
\hline
\end{tabular}

amDAS28 and mESR indicate the means of the last 5 values

$C C P$ cyclic citrullinated peptide, $C R P$ C-reactive protein, DAS28 28-joint disease activity score, ESR erythrocyte sedimentation rate, $M T X$ methotrexate, $R A$ rheumatoid arthritis, $R F$ rheumatoid factor

Table 2 Results of cognitive function and depression/anxiety tests in RA patients and controls

\begin{tabular}{|c|c|c|c|c|c|c|c|c|c|}
\hline & $\begin{array}{l}\text { Abnor- } \\
\text { mal }^{\mathrm{a}}(\uparrow \\
\text { or } \downarrow)\end{array}$ & RA (total) & RA (MTX) & RA (biologic) & Controls & $p$ (RA vs control) & $\begin{array}{l}p(\mathrm{MTX} \\
\text { vs con- } \\
\text { trol) }\end{array}$ & $\begin{array}{l}p \text { (biologic } \\
\text { vs control) }\end{array}$ & $\begin{array}{l}p \text { (MTX } \\
\text { vs bio- } \\
\text { logic) }\end{array}$ \\
\hline$n$ & & 60 & 20 & 40 & 39 & & & & \\
\hline \multicolumn{10}{|c|}{ Cognitive function tests } \\
\hline MOCA total $(n)$ & $\downarrow$ & $23.3 \pm 3.8$ & $24.6 \pm 2.2$ & $22.6 \pm 4.2$ & $25.6 \pm 2.4$ & 0.002 & 0.188 & 0.001 & 0.089 \\
\hline TMT-A time (s) & $\uparrow$ & $69.0 \pm 26.3$ & $57.9 \pm 15.4$ & $76.2 \pm 29.7$ & $53.1 \pm 14.3$ & 0.029 & 0.420 & 0.007 & 0.041 \\
\hline TMT-B time (s) & $\uparrow$ & $108.2 \pm 48.4$ & $98.4 \pm 38.9$ & $113.8 \pm 56.7$ & $76.3 \pm 22.7$ & 0.048 & 0.058 & 0.140 & 0.819 \\
\hline VST-A time (s) & $\uparrow$ & $20.6 \pm 9.3$ & $18.8 \pm 7.9$ & $21.6 \pm 9.8$ & $18.0 \pm 10.1$ & 0.040 & 0.450 & 0.019 & 0.232 \\
\hline VST-B time (s) & $\uparrow$ & $23.0 \pm 9.6$ & $20.7 \pm 8.2$ & $24.1 \pm 10.1$ & $19.3 \pm 5.8$ & 0.051 & 0.994 & 0.007 & 0.127 \\
\hline VST-C time (s) & $\uparrow$ & $36.9 \pm 18.1$ & $33.9 \pm 13.9$ & $38.4 \pm 19.9$ & $29.7 \pm 9.3$ & 0.075 & 0.308 & 0.069 & 0.504 \\
\hline WAIS total $(n)$ & $\downarrow$ & $25.4 \pm 13.3$ & $28.0 \pm 14.1$ & $24.1 \pm 12.9$ & $28.5 \pm 12.6$ & 0.003 & 0.094 & 0.006 & 0.455 \\
\hline BVRT total $(n)$ & $\downarrow$ & $6.58 \pm 1.91$ & $6.75 \pm 1.87$ & $6.50 \pm 1.90$ & $7.67 \pm 1.98$ & 0.005 & 0.074 & 0.006 & 0.662 \\
\hline BVRT error $(n)$ & $\uparrow$ & $3.67 \pm 0.93$ & $3.50 \pm 1.15$ & $3.75 \pm 0.81$ & $3.54 \pm 1.07$ & 0.655 & 0.860 & 0.456 & 0.412 \\
\hline \multicolumn{10}{|c|}{ Depression/anxiety tests } \\
\hline STAIS $(n)$ & $\uparrow$ & $45.5 \pm 8.5$ & $43.4 \pm 9.0$ & $46.6 \pm 8.1$ & $36.9 \pm 9.1$ & $<0.001$ & 0.012 & $<0.001$ & 0.134 \\
\hline $\operatorname{STAIT}(n)$ & $\uparrow$ & $48.0 \pm 11.0$ & $46.0 \pm 11.6$ & $48.9 \pm 10.7$ & $41.1 \pm 9.0$ & 0.004 & 0.183 & 0.002 & 0.384 \\
\hline $\mathrm{BDI}(n)$ & $\uparrow$ & $13.2 \pm 8.8$ & $12.3 \pm 9.2$ & $13.6 \pm 8.7$ & $8.9 \pm 6.5$ & 0.023 & 0.251 & 0.015 & 0.432 \\
\hline
\end{tabular}

Significantly different values are in bold italics

$B D I$ Beck depression inventory, BVRT Benton visual retention test, MOCA montreal cognitive assessment test, $M T X$ methotrexate, $R A$ rheumatoid arthritis, STAI state-trait anxiety inventory, TMT trail making test, VST victoria stroop test, WAIS Wechsler Adult Intelligence Scale

${ }^{a}$ Abnormal column indicates whether higher $(\uparrow)$ or lower $(\downarrow)$ values are abnormal

numbers and letters in sequence. The score on each part is the time required to complete the task. The shorter time indicates better function [25].
Executive function The Victoria Stroop Test (VST) is a brief version of the Stroop task. The Stroop task measures the cognitive control by assessing the ease with which a 
person can maintain a goal in mind and suppress a habitual response in favor of a less familiar one (response inhibition). VST contains 24 items in each of the following conditions: naming the color of dots (VST-A), of neutral words (VST-B), and of color words printed in contrasting colors (VST-C). We determined the time in seconds. In general, the VST tests assess executive functions [25]. TMT-B (see above) also assesses executive function [25].

Memory The Benton Visual Retention Test (BVRT) was used to assess the patient's immediate visual memory and visuo-constructive skills. In the BVRT, 10 pictures with geometric figures are shown one after the other and the patient has to draw the designs as accurately as possible. Administration A (10 s exposure to each card with immediate recall by drawing) was used. The total score corresponds to the number of correct reproductions (maximum 10). The error score indicates the number of errors. Evidently, more correct reproductions and less errors indicate intact skills [29].

Anxiety The Spielberger State-Trait Anxiety Inventory (STAI) is a commonly used self-scored measure of trait and state anxiety. It is composed of dot, neutral word and color word tasks. Both the time of task completion and the number of errors have been recorded. STAIS indicates state (current status) and STAIT measures trait (general status) [30]. Lower STAIS or STAIT values represent less trait and state anxiety.

Depression The Beck Depression Inventory (BDI) is a self-report rating inventory that measures characteristic attitudes and symptoms of depression [31]. Higher BDI values represent higher degree of depression.

\section{SF-36 test of general health status}

Short Form 36 (SF-36) measures general health status [32, 33]. The SF-36v2 questionnaire consists of 36 questions and provides scores for 8 individual subscales (weighted sums of the respective questions): physical function (PF), role physical (RP), bodily pain (BP), global health (GH), vitality (VT), social function (SF), role emotional (RE) and mental health $(\mathrm{MH})$. The score range of each scale is between 0 and 100; the lower the score the more disability. Scores for each domain were calculated and were combined to form the physical component summary (PCS; $\mathrm{PF}+\mathrm{RP}+\mathrm{BP}+\mathrm{GH})$ and mental component summary (MCS; VT + SF + RE + MH) scores [32, 33]. In this study, the paper-based, Hungarian validated version of the SF-36v2 was used [24].

\section{Laboratory assessments and disease activity}

Serum IgM RF and high-sensitivity CRP (hsCRP) were assessed by quantitative nephelometry (Cobas Mira Plus, Roche), using RF and CRP reagents, respectively (both
Dialab, Vienna, Austria). RF levels $>50 \mathrm{IU} / \mathrm{ml}$ indicated seropositivity and hsCRP levels $>5 \mathrm{mg} / \mathrm{l}$ were considered elevated. Anti-CCP autoantibodies were detected in serum samples using the second generation Immunoscan-RA CCP2 ELISA test (Euro Diagnostica, Arnhem, The Netherlands). The assay was performed according to the instructions of the manufacturer. A concentration $>25 \mathrm{IU} / \mathrm{ml}$ indicated seropositivity. Erythrocyte sedimentation rate (ESR) was determined by the traditional Westergren method $(\mathrm{mm} / \mathrm{h})$. DAS28-ESR was determined in all RA patients and mean of the last 5 ESR (mESR) and DAS28 values (mDAS28) was also calculated (Table 1).

\section{Transcranial Doppler assessment}

Transcranial Doppler (TCD) assessment of the MCA vessels was performed as described by us before [4]. In brief, MCAs were assessed by TCD using a Multi-Dop T Digital (DWL Compumedics GmbH, Singen, Germany) device. Among TCD indices, CRC reflects the physiological vasoconstrictive and vasodilatory function of precapillary (resistance) arteries. Normally, the increase in CRC after stimuli causing vasodilation is $>30 \%[4,34,35]$. TCD was performed by a single technician (M.S.) and validated by a neuroradiologist (C.O.).

\section{Carotid artery ultrasound examination}

Common carotid artery (CCA) assessments were carried out using a duplex ultrasound system (Vivid E, GE Healthcare) using a $8 \mathrm{MHz}$ linear array transducer (GE Probe 8L-RS) as described by us elsewhere $[4,36]$. Longitudinal highresolution B-mode ultrasound scans were employed over both right and left CCA and were R-synchronized and recorded. Carotid artery intima-media thickness (cIMT; $\mathrm{mm}$ ) was defined as the distance between the first and second echogenic lines from the lumen, taking the average of five measurements on both sides. All visible plaques were also detected. Assessments were performed by a single observer (AS).

\section{Brain MRI investigations}

Altogether 49 out of the 60 RA patients also underwent brain MRI studies (Siemens Magnetom Verio 3T, Siemens, Munich, Germany) to assess focal vascular lesions, emollition and atrophy. All MRI scans were performed by a single radiologist (LK) and also read by a neuroradiologist (CO). Hyperintense lesions detected by $\mathrm{T} 2$ and flair sequences not characteristic for any white matter diseases were considered vascular lesions. A global cortical atrophy score was used as follows: 0 , no atrophy; 1 , mild atrophy with opening of 
sulci; 2 , moderate atrophy with volume loss of gyri; 3 , severe atrophy.

\section{Statistical analysis}

Statistical analysis was processed with IBM SPSS 22 software. Data are expressed as the mean \pm SD and frequencies and percentages. Normality of data was assessed by Kolmogorov-Smirnov test. Continuous variables were compared by Mann-Whitney test. Simple correlations were determined by Spearman's analysis. Multiple linear regression analysis using the backward method was used to determine correlations and independent associations between parameters. Cognitive tests were the dependent variables and several other parameters were independent variables. The $\beta$ standardized linear coefficients showing linear correlations between two parameters were determined. The B (+ 95\% CI) regression coefficient indicated independent association between the dependent and independent variable during changes. $P$ values $<0.05$ were considered significant.

\section{Results}

\section{Basic characteristics of RA patients and controls}

The major characteristics of the patients are shown in Table 1. Their mean age was $60.7 \pm 9.5$ (range $27-78$ ) years and their mean disease duration was $11.6 \pm 7.6$ (range 2-36) years. Altogether $70 \%$ were IgM rheumatoid factor (RF) positive and 65\% were ACPA (anti-CCP2) positive.

The number of "school years" was not different between RA patients and controls. However, it was lower in biologic $(10.8 \pm 3.0$ years $)$ compared to MTX-treated patients $(12.5 \pm 2.7$ years; $p=0.014)$ and controls $(12.3 \pm 1.8$ years; $p=0.004)$ (Table 1).

There were more smokers among the total RA, biologic- and MTX-treated RA groups compared to controls $(p<0.05)$. One-fourth of controls but only $8.3 \%$ of RA patients regularly consumed alcohol $(p<0.05)$ (Table 1$)$.

CCA plaques were detected in 33 RA patients $(55 \%)$. Altogether $46.9 \%$ of RA patients but only $23.5 \%$ of controls had at least one MRI vascular lesion in their left hemisphere $(p=0.030)$. Similarly, 23 patients $(46.9 \%)$, but only $20.6 \%$ of controls had MRI lesions in their right hemisphere $(p=0.014)$. Altogether $26.5 \%$ of RA patients and $2.9 \%$ of controls had cerebral atrophy $(p=0.005)$ (data not shown).

\section{Results of cognitive tests, depression and anxiety inventories}

The MOCA total score was significantly lower (abnormal) in all RA $(p=0.002)$ and in biologic-treated patients $(p=0.001)$ compared to controls (Table 2). MOCA score $<26$ (indicating cognitive impairment) was observed in $63 \%, 50 \%, 70 \%$ and $46 \%$ of patients in the total RA, MTX, biologic sub-groups and controls, respectively (all RA vs controls: $p=0.032$; data not shown).

All $(p=0.029)$ and biologic-treated RA patients $(p=0.007)$ had longer TMT-A times than controls. Biologictreated patients also required longer time vs MTX-treated ones $(p=0.041)$. In TMT-B, RA patients also needed more time than controls $(p=0.048)$ (Table 2$)$.

In VST-A, RA patients $(p=0.040)$ and the biologictreated subset $(p=0.019)$ needed more time to complete the task than controls. In VST-B, biologic-treated RA patients required more time vs controls $(p=0.007)$ (Table 2).

WAIS digit symbol scores were significantly lower (abnormal) in the total $(p=0.003)$ and biologic-treated RA groups $(p=0.006)$ compared to controls (Table 2 ).

In BVRT, the number of correct reproductions was significantly lower in the full RA cohort $(p=0.005)$ and biologictreated patients $(p=0.006)$ compared to controls. There were no differences in BVRT errors (Table 2).

Regarding anxiety/depression tests, STAIS values were significantly higher (abnormal) in all $(p<0.001)$, MTXtreated $(p=0.012)$ and biologic-treated patients $(p<0.001)$ vs controls. STAIT values were also higher in the total $(p=0.004)$ and biologic-treated RA group $(p=0.002)$ vs controls (Table 2). BDI values were significantly higher (abnormal) in all $(p=0.023)$ and biologic-treated patients $(p=0.015)$ vs controls (Table 2). As STAIT and BDI are anxiety/depression tests, we performed an additional analysis to determine the additional value of STAIT and BDI tests in studying cognitive function of RA patients. Here, RA patients with normal performance in all cognitive tests were considered those with "normal cognitive function". If at least one test gave abnormal result, that individual had cognitive abnormality. Based on this assessment, out of the 60 RA patients, 25 had cognitive impairment and 35 had normal cognitive function. When these two RA groups were compared based on STAIT and BDI, those with cognitive impairment have significantly higher STAIT $(p=0.011)$ and BDI $(p=0.012)$ compared to those performing well in all tests. Controls did not have such differences. Thus, depression and anxiety as measured by BDI and STAIT, respectively, can further differentiate between RA patients who had at least one other abnormal test results and those with normal cognitive test performance.

We used data from the previous SF-36 Hungarian population norm study representing women with 58-61 years of age that corresponds to our RA subset [24]. SF-36 scores were lower in RA patients vs controls in all domains except RE. Three out of the four mental domains of the SF-36 (VT, $\mathrm{SF}$ and $\mathrm{MH}$ ) were impaired in RA patients and RA subsets compared to controls $(p<0.05)$ (Fig. 1). 


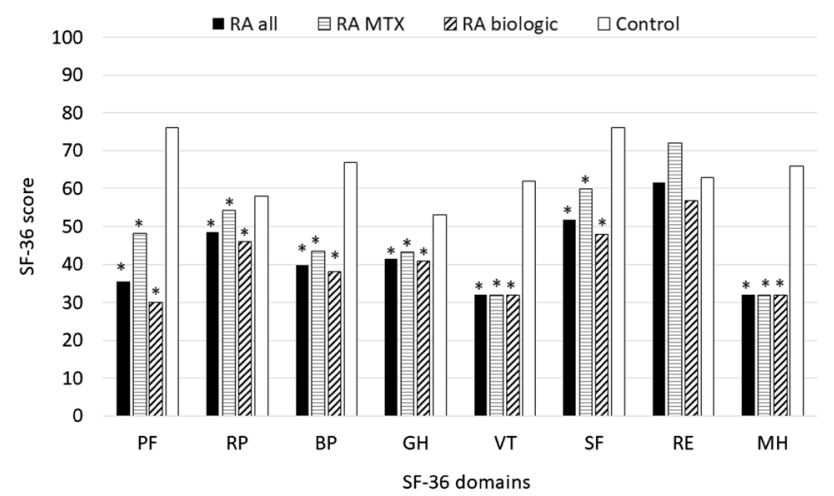

${ }^{*} \mathrm{p}<0.05$ vs control

Fig. 1 Results of the SF-36 test in all, methotrexate (MTX)- and biologic-treated rheumatoid arthritis (RA) patients and controls. The 8 domains are: physical function (PF), role physical (RP), bodily pain (BP), global health $(\mathrm{GH})$, vitality (VT), social function (SF), role emotional (RE) and mental health (MH)

\section{Correlations among cognitive function tests in RA patients}

All cognitive function tests were correlated with each other in RA patients. As many of them assess somewhat overlapping characteristics, numerous correlations could be identified. In general, most cognitive test results correlated with many others (data not shown).

\section{Correlations between cognitive function and SF-36 in RA patients}

STAIS, STAIT and BDI values showed significant inverse correlations with all 8 SF-36 domains, as well as with MCS $(p<0.05)$. WAIS Digit Symbol values exerted positive correlations with 7 SF-36 domains, PCS and MCS $(p<0.05)$ (data not shown).

\section{Correlations between cognitive function and other parameters in RA patients}

Longer TMT-A, TMT-B, VST-A, VST-B, VST-C times, more BVRT errors and lower WAIS scores correlated with age $(p<0.05)$. Longer VST-A time correlated with disease duration $(p=0.027)$, while STAIT correlated with mESR $(p=0.006)$ and mDAS28 $(p=0.018)$. MOCA, STAIS, STAIT, BDI values, VST-A, VST-B and VST-C times, WAIS and BVRT total scores and BVRT error numbers showed associations with years in education $(p<0.05)$ (Table 3).

\section{Associations between cognitive function and vascular abnormalities in RA patients}

Left MCA CRC positively correlated with MOCA $(p=0.016)$ and inversely with BDI $(p=0.008)$. Right MCA CRC exerted positive correlation with BVRT total values $(p=0.033)$. Right cIMT inversely correlated with WAIS Digit Symbol scores $(p=0.024)$ (Table 3). RA patients with right carotid plaques had higher STAIS, STAIT values, longer VST-C times and lower WAIS scores $(p<0.05)$. Patients with left carotid plaques had higher STAIS, STAIT, BDI scores, longer TMT-A and VST-C times, and lower WAIS scores $(p<0.05)$ (Table 4$)$. Patients with MRI vascular lesions had longer VST-A times $(p=0.032)$, while those with brain atrophy had lower WAIS scores (Table 4).

\section{Determinants of cognitive function in RA patients}

Univariate and multiple regression analyses were performed to assess independent determinants of cognitive function (Table 5).

In the univariate analysis, numerous tests correlated with age and/or school years $(p<0.05)$. STAIS, STAIT, TMT-A time, VST-C time and WAIS Digit Symbol score were associated with the presence of carotid plaques $(p<0.05)$. WAIS scores also correlated with right cIMT $(p=0.024)$. VST-A time correlated with the presence of MRI vascular lesions $(p=0.006)$. BDI and BVRT total scores associated with left and right MCA CRC, respectively $(p<0.05)$. STAIT correlated with mESR $(p=0.009)$ and mDAS28 $(p=0.02)$. Biologic- vs MTX-treated patients had longer TMT-A times $(p=0.03)$ (Table 5).

In the multiple regression analysis of RA patients, age was independent predictor of TMT-A time $(p=0.001)$, VST-B time $(p=0.005)$, VST-C time $(p=0.001)$ and WAIS scores $(p=0.001)$. School years determined MOCA $(p=0.006)$, STAIS $(p<0.001)$, BDI $(p=0.001)$, BVRT total scores $(p<0.001)$ and errors $(p<0.001)$. The presence of left carotid plaques determined STAIT $(p=0.036)$, while that of MRI lesions predicted longer VST-A times $(p=0.001)$. The use of biologics vs MTX was an independent predictor of TMT-A times $(p=0.016)$ (Table 5).

\section{Discussion}

We cross-sectionally assessed cognitive functions and depression/anxiety in female RA patients compared to gender- and age-matched healthy individuals using standard validated measures. Moreover, we aimed to explore associations 
Table 3 Significant correlations between cognitive test results and other parameters in RA patients

\begin{tabular}{|c|c|c|c|c|c|c|c|c|c|}
\hline & $\begin{array}{l}\text { Abnor- } \\
\mathrm{mal}^{\mathrm{a}}(\uparrow \\
\text { or } \downarrow)\end{array}$ & Age & Disease duration & $\mathrm{mESR}^{\mathrm{b}}$ & mDAS28b & School years & CRC (left) & CRC (right) & cIMT (right) \\
\hline \multicolumn{10}{|c|}{ Cognitive function tests } \\
\hline $\operatorname{MOCA}(n)$ & $\downarrow$ & & & & & $\begin{array}{l}R=0.334 \\
p=0.009\end{array}$ & $\begin{array}{l}R=0.445 \\
p=0.016\end{array}$ & & \\
\hline TMT-A time (s) & $\uparrow$ & $\begin{array}{l}R=0.525 \\
p=0.002\end{array}$ & & & & & & & \\
\hline TMT-B time (s) & $\uparrow$ & $\begin{array}{l}R=0.492 \\
p=0.013\end{array}$ & & & & & & & \\
\hline VST-A time (s) & $\uparrow$ & $\begin{array}{l}R=0.366 \\
p=0.004\end{array}$ & $\begin{array}{l}R=0.285 \\
p=0.027\end{array}$ & & & $\begin{array}{l}R=-0.318 \\
p=0.013\end{array}$ & & & \\
\hline VST-B time (s) & $\uparrow$ & $\begin{array}{l}R=0.424 \\
p=0.001\end{array}$ & & & & $\begin{array}{l}R=-0.398 \\
p=0.002\end{array}$ & & & \\
\hline VST-C time (s) & $\uparrow$ & $\begin{array}{l}R=0.486 \\
p<0.001\end{array}$ & & & & $\begin{array}{l}R=-0.345 \\
p=0.007\end{array}$ & & & \\
\hline WAIS total $(n)$ & $\downarrow$ & $\begin{array}{l}R=-0.311 \\
p=0.001\end{array}$ & & & & $\begin{array}{l}R=0.456 \\
p<0.001\end{array}$ & & & $\begin{array}{l}R=-0.291 \\
p=0.024\end{array}$ \\
\hline BVRT total $(n)$ & $\downarrow$ & & & & & $\begin{array}{l}R=0.378 \\
p=0.003\end{array}$ & & $\begin{array}{l}R=0.342 \\
p=0.033\end{array}$ & \\
\hline BVRT error $(n)$ & $\uparrow$ & $\begin{array}{l}R=0.290 \\
p=0.025\end{array}$ & & & & $\begin{array}{l}R=-0.369 \\
p=0.004\end{array}$ & & & \\
\hline \multicolumn{10}{|c|}{ Depression/anxiety tests } \\
\hline STAIS $(n)$ & $\uparrow$ & & & & & $\begin{array}{l}R=-0.489 \\
p<0.001\end{array}$ & & & \\
\hline STAIT $(n)$ & $\uparrow$ & & & $\begin{array}{l}R=0.354 \\
p=0.006\end{array}$ & $\begin{array}{l}R=0.307 \\
p=0.018\end{array}$ & $\begin{array}{l}R=-0.368 \\
p=0.004\end{array}$ & & & \\
\hline $\mathrm{BDI}(n)$ & $\uparrow$ & & & & & $\begin{array}{l}R=-0.425 \\
p=0.001\end{array}$ & $\begin{array}{l}R=-0.481 \\
p=0.008\end{array}$ & & \\
\hline
\end{tabular}

$B D I$ Beck depression inventory, BVRT Benton visual retention test, $c I M T$ carotid intima-media thickness, $C R C$ circulatory reserve capacity, DAS28 28-joint disease activity score, ESR erythrocyte sedimentation rate, MOCA Montreal Cognitive Assessment test, STAI state-trait anxiety inventory, TMT Trail Making Test, VST Victoria Stroop Test, WAIS Wechsler Adult Intelligence Scale

${ }^{a}$ Abnormal column indicates whether higher $(\uparrow)$ or lower $(\downarrow)$ values are abnormal

${ }^{\mathrm{b}} \mathrm{mDAS} 28$ and mESR indicate the means of the last 5 values

between cognitive decline and RA-related parameters, CV and cerebrovascular pathophysiology, quality of life and therapy. Among the domains of cognitive function $[9,10]$, we assessed attention/concentration (WAIS, TMT-A, TMTB), memory (BVRT), executive functions (TMT-B, VST). We applied MOCA as a screening tool. We also compared cognitive dysfunction with depression (BDI) and anxiety (STAI). The novelty of our study is that we used many tests of cognitive function and we associated these tests with cerebrovascular imaging including TCD, carotid ultrasound and brain MRI.

MOCA score, TMT-A, TMT-B, VST-A and VST-B times, WAIS Digit Symbol scores and the number of correct reproductions in BVRT were significantly abnormal in RA patients vs controls. Our results confirm that RA is associated with cognitive impairment $[9,11,13]$.

As different domains of cognitive function are interrelated $[9,10]$, it is not surprising that results of the various cognitive function tests correlated with each other in RA. Depression and anxiety are common among RA patients [6, 7]; however, some domains of cognitive dysfunction did not correlate with BDI or STAI suggesting that cognitive decline in RA also involves other factors. Although cognitive function and depression/anxiety have been tested in the same RA patients [15, 37], we did not find any extensive studies correlating these entities.

Three out of 4 SF-36 mental domains were impaired in RA patients. The WAIS screening test showed association with 7 out of 8 SF-36 domains. TMT-A and TMT-B times also correlated with some, mainly physical SF-36 domains. Moreover, STAIS, STAIT and BDI values were associated with all 8 SF-36 domains suggesting that, in RA, both cognitive dysfunction and depression/anxiety may have negative impact on the general health status of the patients. We have found only one study that analyzed the associations of cognitive function with SF-36 in RA. Kim et al. [38] 


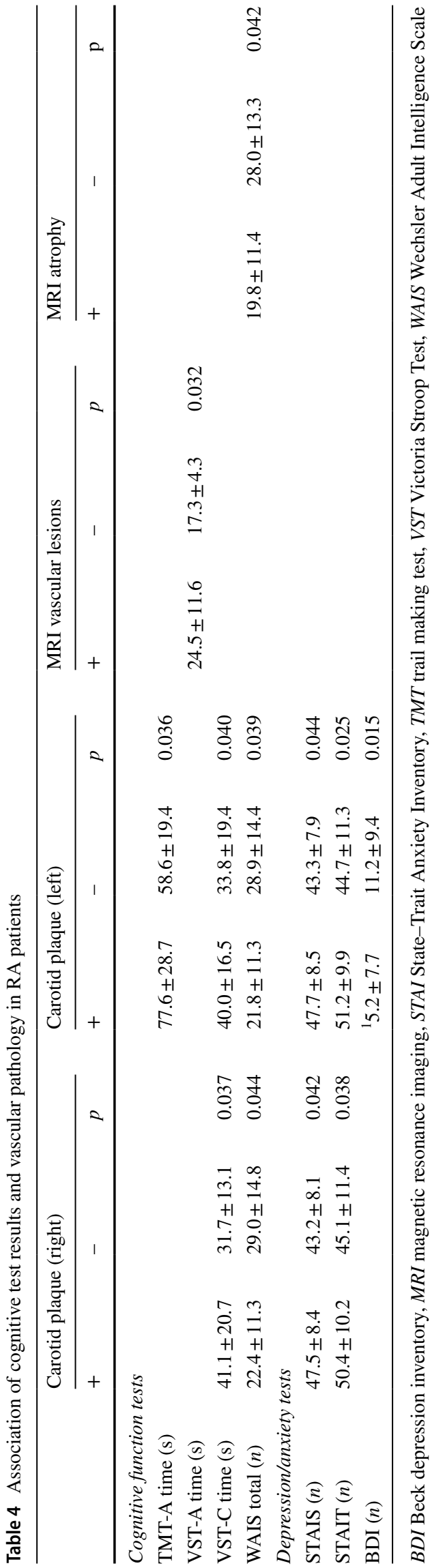

reported an independent association of MCS with MOCA. Shin et al. [11] used 16 cognitive indices and found relationship between cognitive and functional limitations. However, studies exploring the impact of cognitive impairment on health-related quality of life in RA are, on the whole, scarce and deserve further investigation.

In our RA cohort, cognitive function tests were also correlated with other parameters, such as age, disease duration, school years, mESR or mDAS28. Shin et al. [13] and Lee et al. [39] also associated cognitive impairment with low education and disease activity, respectively.

As atherosclerosis has been implicated in cognitive impairment in RA [9, 13], we also determined cerebral vascular lesions and atrophy, carotid atherosclerosis and CRC. We indeed found correlations between these vascular pathologic alterations and cognitive dysfunction. As described above, functional MRI [40] showed pathological cerebral alterations in RA. In non-RA, stroke patients, other investigators also found associations between cognitive decline and carotid atherosclerosis [41]. Cerebral blood flow in association with cognitive function has not yet been assessed in RA before. In non-RA studies, cerebral arterial circulation was associated with cognitive impairment [42, 43].

RA patients treated with biologics usually resemble a more severe form of disease than those controlled by MTX only. In our study, TMT-A times were longer in biologicvs MTX-treated patients. Although some studies suggest that MTX may itself cause cognitive impairment [20], it is possible that the severity of RA may even be more important in this respect. Meade et al. [20] reported that patients taking higher doses of MTX have lower cognitive performance; however, it was still in the normal range. They also emphasized that due to the various treatment protocols, one needs to be cautious when drawing conclusions on the possible effects of anti-rheumatic drugs on cognition [9, 20]. Although sustained inflammation may be involved in cognitive decline in RA [9, 17], only very few studies evaluated the longitudinal effects of anti-rheumatic drugs on cognitive function. In the open-label study of Raftery et al. [22], adalimumab improved full scale, verbal and performance IQ in parallel with improvements of DAS28. Camara et al. [44] reported that centrally administered etanercept improved anxiety-like behavior in mice. Khandaker et al. [23] presented a protocol of a trial initiated in 2018, where behavioral and cognitive measures will be evaluated in tocilizumabtreated patients. In addition to pharmacotherapy, tailored cognitive behavioral therapy introduced in early RA was found to be beneficial in various studies $[45,46]$.

Our study may have strengths and limitations. Our study is rather comprehensive applying numerous different cognitive function tests in the context of clinical, laboratory factors, brain vascular lesions, carotid atherosclerosis, CRC and quality of life. The limitations of our study include 
Table 5 Linear regression analysis of cognitive function test results and other parameters in RA patients

\begin{tabular}{|c|c|c|c|c|c|c|c|c|c|}
\hline \multirow[t]{2}{*}{ Dependent variable } & \multirow{2}{*}{$\begin{array}{l}\text { Independent vari- } \\
\text { able }\end{array}$} & \multicolumn{4}{|l|}{ Univariate } & \multicolumn{4}{|l|}{ Multiple } \\
\hline & & $B$ & $95 \% \mathrm{CI}$ & $\beta$ & $p$ & $B$ & $95 \% \mathrm{CI}$ & $\beta$ & $p$ \\
\hline \multicolumn{10}{|c|}{ Cognitive function tests } \\
\hline MOCA total & School years & 0.446 & 0.133 to 0.760 & 0.350 & 0.006 & 0.446 & 0.133 to 0.760 & 0.350 & 0.006 \\
\hline \multirow[t]{3}{*}{ TMT-A time } & Age & 1.763 & 0.717 to 2.809 & 0.525 & 0.002 & 1.798 & 0.831 to 2.764 & 0.536 & 0.001 \\
\hline & Biologic vs MTX & 6.076 & -0.012 to 12.163 & 0.343 & 0.030 & 6.345 & 1.252 to 11.432 & 0.359 & 0.016 \\
\hline & $\begin{array}{l}\text { Carotid plaque } \\
\text { (left) }\end{array}$ & 9.506 & 0.623 to 18.388 & 0.365 & 0.037 & 4.344 & -3.891 to 12.580 & 0.167 & 0.290 \\
\hline TMT-B time & Age & 3.007 & 0.949 to 5.064 & 0.492 & 0.006 & 3.007 & 0.949 to 5.064 & 0.492 & 0.006 \\
\hline \multirow[t]{2}{*}{ VST-A time } & Disease duration & 0.341 & 0.037 to 0.645 & 0.282 & 0.029 & 0.256 & -0.108 to 0.619 & 0.193 & 0.163 \\
\hline & $\begin{array}{l}\text { MRI vascular } \\
\text { lesions }\end{array}$ & 3.572 & 1.103 to 6.041 & 0.398 & 0.006 & 4.362 & 1.827 to 6.897 & 0.476 & 0.001 \\
\hline \multirow[t]{2}{*}{ VST-B time } & Age & 0.308 & 0.058 to 0.559 & 0.308 & 0.017 & 0.493 & 0.155 to 0.830 & 0.385 & 0.005 \\
\hline & School years & -0.911 & -1.722 to -0.100 & -0.283 & 0.028 & -0.731 & -1.539 to 0.078 & -0.227 & 0.076 \\
\hline \multirow[t]{3}{*}{ VST-C time } & Age & 0.819 & 0.369 to 1.268 & 0.432 & 0.001 & 0.819 & 0.369 to 1.268 & 0.432 & 0.001 \\
\hline & School years & -1.717 & -3.252 to -0.181 & -0.282 & 0.029 & -0.751 & -2.004 to 1.101 & -0.114 & 0.418 \\
\hline & $\begin{array}{l}\text { Carotid plaque } \\
\text { (right) }\end{array}$ & 4.709 & 0.119 to 9.298 & 0.260 & 0.045 & 1.414 & -3.256 to 6.084 & 0.078 & 0.547 \\
\hline \multirow[t]{4}{*}{ WAIS total } & Age & -0.573 & -0.907 to -0.239 & -0.411 & 0.001 & -1.098 & -1.705 to -0.491 & -0.604 & 0.001 \\
\hline & School years & 2.042 & 0.995 to 3.089 & 0.456 & $<0.001$ & 0.574 & -0.756 to 1.904 & 0.138 & 0.386 \\
\hline & cIMT (right) & -144.84 & $\begin{array}{c}-269.887 \text { to } \\
19.792\end{array}$ & -0.291 & 0.024 & -93.847 & $\begin{array}{c}-204.589 \text { to } \\
16.895\end{array}$ & -0.189 & 0.095 \\
\hline & $\begin{array}{l}\text { Carotid plaque } \\
\text { (left) }\end{array}$ & -3.533 & -6.883 to -0.183 & -0.267 & 0.039 & -1.329 & -4.501 to 1.844 & -0.100 & 0.405 \\
\hline \multirow[t]{2}{*}{ BVRT total } & School years & 0.286 & 0.142 to 0.430 & 0.372 & $<0.001$ & 0.286 & 0.142 to 0.430 & 0.372 & $<0.001$ \\
\hline & MCA CRC (right) & 0.050 & 0.002 to 0.097 & 0.329 & 0.041 & 0.043 & 0.008 to 0.088 & 0.312 & 0.061 \\
\hline \multirow[t]{2}{*}{ BVRT error } & Age & 0.029 & 0.006 to 0.051 & 0.247 & 0.014 & 0.019 & -0.006 to 0.043 & 0.192 & 0.129 \\
\hline & School years & -0.132 & -0.204 to -0.060 & -0.348 & $<0.001$ & -0.132 & -0.204 to -0.060 & -0.348 & $<0.001$ \\
\hline \multicolumn{10}{|c|}{ Depression/anxiety tests } \\
\hline \multirow[t]{3}{*}{ STAIS } & School years & -1.410 & -2.057 to -0.763 & -0.497 & $<0.001$ & -1.410 & -2.057 to -0.763 & -0.497 & $<0.001$ \\
\hline & $\begin{array}{l}\text { Carotid plaque } \\
\text { (right) }\end{array}$ & 2.168 & 0.027 to 4.303 & 0.257 & 0.047 & 0.842 & -1.255 to 2.940 & 0.100 & 0.425 \\
\hline & $\begin{array}{l}\text { Carotid plaque } \\
\text { (left) }\end{array}$ & 2.200 & 0.073 to 4.327 & 0.262 & 0.043 & 1.052 & -1.022 to 3.126 & 0.125 & 0.314 \\
\hline \multirow[t]{4}{*}{ STAIT } & School years & -1.295 & -2.205 to -0.384 & -0.350 & 0.006 & -0.887 & -1.800 to 0.026 & -0.242 & 0.057 \\
\hline & $\mathrm{mESR}^{\mathrm{a}}$ & 0.290 & 0.076 to 0.503 & 0.338 & 0.009 & 0.057 & -2.980 to 0.413 & 0.076 & 0.749 \\
\hline & $\operatorname{mDAS} 28^{\mathrm{a}}$ & 4.482 & 0.725 to 8.239 & 0.302 & 0.020 & 1.207 & -3.090 to 5.503 & 0.089 & 0.576 \\
\hline & $\begin{array}{l}\text { Carotid plaque } \\
\text { (left) }\end{array}$ & 3.267 & 0.525 to 6.008 & 0.299 & 0.020 & 3.480 & 0.247 to 6.713 & 0.318 & 0.036 \\
\hline \multirow[t]{2}{*}{ BDI } & School years & -1.217 & -1.922 to -0.511 & -0.413 & 0.001 & -1.217 & -1.922 to -0.511 & -0.413 & 0.001 \\
\hline & MCA CRC (left) & -0.246 & -0.474 to -0.019 & -0.393 & 0.035 & 0.219 & -0.455 to 0.016 & -0.426 & 0.065 \\
\hline
\end{tabular}

Linear regression, backward method, $\beta$ standardized linear coefficient, $B(+95 \% C I)$ regression coefficient. See text for abbreviations and explanations

${ }^{a}$ mDAS28 and mESR indicate the means of the last 5 values

the relatively low number of patients and healthy controls matched for many but not all potentially relevant characteristics. Hence, we suggest to consider our results as explorative rather than conclusive and further studies involving larger samples, representative for the RA population are encouraged. However, feasibility issues may arise on performing imaging technologies alongside the standard cognitive tests in large groups of patients and controls, one of the strengths of our study. Given the cross-sectional design, the effect of anti-rheumatic therapy on cognitive function could not be longitudinally analyzed; this is an important area for further research. While laboratory biomarkers and quality of life could be numerically determined, alcohol intake, smoking habits and education were rather estimated. Applying 
measures and categories that allow comparisons with population norms (e.g., European Health Survey) for alcohol and tobacco consumption as well as for educational levels (ISCED categories) is suggested in further studies. Finally, we were unable to perform Bonferroni corrections.

In conclusion, our study suggests that RA patients had significant cognitive impairment as determined by multiple tests. Various domains of cognitive dysfunction may be associated with age, lower education, disease duration and activity, inflammatory markers, primarily the mental components of SF-36 and some measures of vascular pathology. From the practical point of view, RA patients, especially those with higher age, lower education, sustained disease activity, should be evaluated for cognitive dysfunction by any tests used in our study and also by others. Although some anti-rheumatic agents may impair cognitive function, the control of inflammation by MTX and biologics in association with tailored behavioral-cognitive therapies may benefit our RA patients.

Acknowledgements Open access funding provided by University of Debrecen (DE). This research was supported by the European Union and the State of Hungary and co-financed by the European Social Fund in the framework of TAMOP-4.2.4.A/2-11/1-2012-0001 'National Excellence Program' (ZS); as well as by the European Union grants GINOP-2.3.2-15-2016-00015 and GINOP-2.3.2-15-2016-00050 (ZS).

\section{Compliance with ethical standards}

Conflicts of interest None of the authors have any potential conflicts of interest.

Research involving human participants This research involved human participants.

Ethical approval Ethical approval (No. 1046-63/2015) was obtained from the Regional/Institutional Review Board of Miskolc University. The study was performed according to the Declaration of Helsinki.

Informed consent All patients and controls signed informed consent.

Open Access This article is distributed under the terms of the Creative Commons Attribution 4.0 International License (http://creativeco mmons.org/licenses/by/4.0/), which permits unrestricted use, distribution, and reproduction in any medium, provided you give appropriate credit to the original author(s) and the source, provide a link to the Creative Commons license, and indicate if changes were made.

\section{References}

1. Symmons DP (2002) Epidemiology of rheumatoid arthritis: determinants of onset, persistence and outcome. Best Pract Res Clin Rheumatol 16:707-722

2. Joaquim AF, Appenzeller S (2015) Neuropsychiatric manifestations in rheumatoid arthritis. Autoimmun Rev 14:1116-1122

3. Meune C, Touze E, Trinquart L, Allanore Y (2010) High risk of clinical cardiovascular events in rheumatoid arthritis: levels of associations of myocardial infarction and stroke through a systematic review and meta-analysis. Arch Cardiovasc Dis 103:253-261

4. Olah C, Kardos Z, Sepsi M, Sas A, Kostyal L, Bhattoa HP et al (2017) Assessment of intracranial vessels in association with carotid atherosclerosis and brain vascular lesions in rheumatoid arthritis. Arthritis Res Ther 19:213

5. Schrepf A, Kaplan CM, Ichesco E, Larkin T, Harte SE, Harris RE et al (2018) A multi-modal mri study of the central response to inflammation in rheumatoid arthritis. Nat Commun 9:2243

6. Fiest KM, Hitchon CA, Bernstein CN, Peschken CA, Walker JR, Graff LA et al (2017) Systematic review and meta-analysis of interventions for depression and anxiety in persons with rheumatoid arthritis. J Clin Rheumatol 23:425-434

7. VanDyke MM, Parker JC, Smarr KL, Hewett JE, Johnson GE, Slaughter JR et al (2004) Anxiety in rheumatoid arthritis. Arthritis Rheum 51:408-412

8. Palkonyai E, Kolarz G, Kopp M, Bogye G, Temesvari P, Palkonyay L et al (2007) Depressive symptoms in early rheumatoid arthritis: a comparative longitudinal study. Clin Rheumatol 26:753-758

9. Meade T, Manolios N, Cumming SR, Conaghan PG, Katz P (2018) Cognitive impairment in rheumatoid arthritis: a systematic review. Arthritis Care Res (Hoboken) 70:39-52

10. Lezak M, Howieson DB, Bigler ED, Tranel D (2012) Neuropsychological assessment, 5th edn. Oxford University Press, New York

11. Shin SY, Julian L, Katz P (2013) The relationship between cognitive function and physical function in rheumatoid arthritis. J Rheumatol 40:236-243

12. Shin SY, Katz P, Julian L (2013) Relationship between perceived cognitive dysfunction and objective neuropsychological performance in persons with rheumatoid arthritis. Arthritis Care Res (Hoboken) 65:481-486

13. Shin SY, Katz P, Wallhagen M, Julian L (2012) Cognitive impairment in persons with rheumatoid arthritis. Arthritis Care Res (Hoboken) 64:1144-1150

14. Abeare CA, Cohen JL, Axelrod BN, Leisen JC, Mosley-Williams A, Lumley MA (2010) Pain, executive functioning, and affect in patients with rheumatoid arthritis. Clin J Pain 26:683-689

15. Appenzeller S, Bertolo MB, Costallat LT (2004) Cognitive impairment in rheumatoid arthritis. Methods Find Exp Clin Pharmacol 26:339-343

16. Shin SY (2012) The relationship between cognitive and physical function in older adults with rheumatoid arthritis: a literature review. J Gerontol Nursing 38:33-42

17. Gorelick PB (2010) Role of inflammation in cognitive impairment: results of observational epidemiological studies and clinical trials. Ann N Y Acad Sci 1207:155-162

18. Agca R, Heslinga SC, Rollefstad S, Heslinga M, McInnes IB, Peters MJ et al (2017) Eular recommendations for cardiovascular disease risk management in patients with rheumatoid arthritis and other forms of inflammatory joint disorders: 2015/2016 update. Ann Rheum Dis 76:17-28

19. Szekanecz Z, Kerekes G, Der H, Sandor Z, Szabo Z, Vegvari A et al (2007) Accelerated atherosclerosis in rheumatoid arthritis. Ann N Y Acad Sci 1108:349-358

20. Meade T, Cumming S, Hallab L, Spencer D, Howe G, Manolios N (2013) A preliminary investigation of cognitive function in rheumatoid arthritis patients on long-term methotrexate treatment. J Health Psychol 18:1353-1359

21. Coluccia D, Wolf OT, Kollias S, Roozendaal B, Forster A, de Quervain DJ (2008) Glucocorticoid therapy-induced memory deficits: acute versus chronic effects. J Neurosci 28:3474-3478

22. Raftery G, He J, Pearce R, Birchall D, Newton JL, Blamire AM et al (2012) Disease activity and cognition in rheumatoid arthritis: an open label pilot study. Arthritis Res Ther 14:R263 
23. Khandaker GM, Oltean BP, Kaser M, Dibben CRM, Ramana R, Jadon DR et al (2018) Protocol for the insight study: a randomised controlled trial of single-dose tocilizumab in patients with depression and low-grade inflammation. BMJ Open 8:e025333

24. Czimbalmos A, Nagy Z, Varga Z, Husztik P (1999) Patient satuisfaction survey by sf-36 questionnaire: determination of hungarian normal values (hungarian). Nepegeszsegugy (Public Health) 80:4-19

25. Strauss E, Sherman EM, Spreen O. A compendium of neuropsychological tests: Administration, norms, and commentary. American chemical society. 2006

26. Nasreddine ZS, Phillips NA, Bedirian V, Charbonneau S, Whitehead V, Collin I et al (2005) The montreal cognitive assessment, moca: a brief screening tool for mild cognitive impairment. J Am Geriatr Soc 53:695-699

27. Wechsler D (1981) The wechsler adult intelligence scale-revised. The Psychological Corporation, New york

28. Szakács F. [types of deficits in intelligence]. Budapest: Medicina. 1995

29. Sivan A (1992) Benton visual retention test, 5th edn. The Psychological Corporation, San antonio

30. Spielberger C (1989) State-trait anxiety inventory: bibliography, 2nd edn. Consulting Psychologists Press, Palo Alto

31. Beck AT, Ward CH, Mendelson M, Mock J, Erbaugh J (1961) An inventory for measuring depression. Arch Gen Psychiatry 4:561-571

32. Matcham F, Scott IC, Rayner L, Hotopf M, Kingsley GH, Norton $S$ et al (2014) The impact of rheumatoid arthritis on quality-of-life assessed using the sf-36: a systematic review and meta-analysis. Semin Arthritis Rheum 44:123-130

33. https://campaign.Optum.Com/content/optum/en/optum-outcomes/ what-we-do/health-surveys.Html

34. Geoepidemiology Ehrenfeld M (2010) The environment and spondyloarthropathies. Autoimmun Rev 9:A325-A329

35. Pindzola RR, Balzer JR, Nemoto EM, Goldstein S, Yonas H (2001) Cerebrovascular reserve in patients with carotid occlusive disease assessed by stable xenon-enhanced ct cerebral blood flow and transcranial doppler. Stroke 32:1811-1817

36. Kerekes G, Szekanecz Z, Der H, Sandor Z, Lakos G, Muszbek L et al (2008) Endothelial dysfunction and atherosclerosis in rheumatoid arthritis: a multiparametric analysis using imaging techniques and laboratory markers of inflammation and autoimmunity. J Rheumatol 35:398-406
37. Tomasevic-Todorovic S, Boskovic K, Filipovic D (2011) Naumovic $\mathrm{N}$ [assessment of memory in patients with rheumatoid arthritis]. Vojnosanit Pregl 68:481-488

38. Kim SH, Kim H, Kim S, Paek S, Koh JH, Lee J et al (2018) Sleep quality independently affects health-related quality of life and cognitive function in korean female patients with rheumatoid arthritis: a case-control study. J Korean Med Sci 33:e216

39. Lee JH, Kim GT, Kim YK, Lee SG (2018) Cognitive function of patients with rheumatoid arthritis is associated with disease activity but not carotid atherosclerotic changes. Clin Exp Rheumatol $36: 856-861$

40. Rech J, Hess A, Finzel S, Kreitz S, Sergeeva M, Englbrecht M et al (2013) Association of brain functional magnetic resonance activity with response to tumor necrosis factor inhibition in rheumatoid arthritis. Arthritis Rheum 65:325-333

41. Yue W, Wang A, Liang H, Hu F, Zhang Y, Deng M et al (2016) Association between carotid intima-media thickness and cognitive impairment in a chinese stroke population: a cross-sectional study. Sci Rep 6:19556

42. Wang X, Jackson DC, Varghese T, Mitchell CC, Hermann BP, Kliewer MA et al (2014) Correlation of cognitive function with ultrasound strain indices in carotid plaque. Ultrasound Med Biol 40:78-89

43. Lim JS, Lee JY, Kwon HM, Lee YS (2017) The correlation between cerebral arterial pulsatility and cognitive dysfunction in alzheimer's disease patients. J Neurol Sci 373:285-288

44. Camara ML, Corrigan F, Jaehne EJ, Jawahar MC, Anscomb H, Baune BT (2015) Effects of centrally administered etanercept on behavior, microglia, and astrocytes in mice following a peripheral immune challenge. Neuropsychopharmacology 40:502-512

45. Sharpe L, Sensky T, Timberlake N, Ryan B, Brewin CR, Allard S (2001) A blind, randomized, controlled trial of cognitive-behavioural intervention for patients with recent onset rheumatoid arthritis: preventing psychological and physical morbidity. Pain 89:275-283

46. Evers AW, Kraaimaat FW, van Riel PL, de Jong AJ (2002) Tailored cognitive-behavioral therapy in early rheumatoid arthritis for patients at risk: a randomized controlled trial. Pain 100:141-153

Publisher's Note Springer Nature remains neutral with regard to jurisdictional claims in published maps and institutional affiliations.

\section{Affiliations}

\section{Csaba Oláh ${ }^{1} \cdot$ Zsófia Kardos $^{2} \cdot$ Mónika Andrejkovics $^{3,4}$ - Enikő Szarka ${ }^{1} \cdot$ Katalin Hodosi ${ }^{5} \cdot$ Andrea Domján $^{5}$. Mariann Sepsi ${ }^{6} \cdot$ Attila Sas $^{7} \cdot$ László Kostyál $^{6} \cdot$ Katalin Fazekas $^{2} \cdot$ Ágnes Flórián ${ }^{2} \cdot$ Katalin Lukács $^{2} \cdot$ Ágnes Miksi $^{2}$. Zsuzsanna Baráth $^{2}$. György Kerekes ${ }^{8}$. Márta Péntek ${ }^{9,10}$. Attila Valikovics ${ }^{7}$ - László Tamási ${ }^{2}$. Dániel Bereczki ${ }^{11}$. Zoltán Szekanecz ${ }^{5}$}

1 Department of Neurosurgery, Borsod County Teaching Hospital, Miskolc, Hungary

2 Department of Rheumatology, Borsod County Teaching Hospital, Miskolc, Hungary

3 Institute of Behavioural Sciences, Faculty of Public Health, University of Debrecen, Debrecen, Hungary

4 Department of Oncoradiology, Szabolcs-Szatmár-Bereg County Hospitals, Jósa András Teaching Hospital, Nyíregyháza, Hungary
5 Department of Rheumatology, Faculty of Medicine, University of Debrecen, Debrecen, Hungary

6 Department of Radiology, Borsod County Teaching Hospital, Miskolc, Hungary

7 Department of Neurology, Borsod County Teaching Hospital, Miskolc, Hungary

8 Department of Angiology, Faculty of Medicine, University of Debrecen, Debrecen, Hungary 
9 Department of Health Economics, Corvinus University, Budapest, Hungary

10 Department of Rheumatology, Flór Ferenc County Hospital, Kistarcsa, Hungary
11 Department of Neurology, Semmelweis University, Budapest, Hungary 